\title{
FIB, SEM, and TEM of Bone/Dental Implant Interfaces
}

\author{
Lucille A. Giannuzzi*, Nicholas J. Giannuzzi**, and Mario J. Capuano*** \\ *FEI Company, 5350 NE Dawson Creek Drive, Hillsboro, OR 97124 USA \\ **Nicholas J. Giannuzzi DDS, 10 Hunter Ave., Miller Place, NY 11764 USA \\ ***Li Oral and Maxillofacial Surgery, 248 Middle Country Road, Selden, NY 11784 USA
}

Typical scanning electron microscopy (SEM) studies of bone/implant systems are performed using top-down approaches. However, understanding the osseointegration mechanism is better performed by analyzing the system in cross-section. In particular, transmission electron microscopy (TEM) has been used to observe the bone/implant interface in cross-section from specimens prepared using ultramicrotomy techniques [1]. However, microtomy can impart large shear stresses, and hence, TEM results often show large amounts of mechanical damage to the specimen. In addition, it is difficult to obtain an electron transparent portion of the ceramic coated metal alloy implant using microtomy techniques. Hence, thin TEM cross-sections encompassing the bone, coating, and implant substrate in its entirety are difficult to obtain.

The focused ion beam (FIB) instrument has been used for well over a decade now to site-specifically cross-section a wide variety of samples in both the physical and the biological sciences [2]. By incorporating a FIB column and an SEM column on the same platform (e.g., a DualBeam instrument) any sample can be cross-sectioned with the FIB and directly analyzed with the SEM and associated techniques. In addition, FIB processes can be directly monitored in real-time using SEM imaging, and site specific in-situ lift-out techniques can be used for TEM specimen preparation.

A Ti-plasma-sprayed $\mathrm{P}$ and $\mathrm{O}$ containing coated Ti dental implant that deemed to have failed was surgically removed from a patient and analyzed using an FEI Quanta 3D DualBeam, a Nova NanoLab DualBeam, and a Tecnai F20 (S)TEM operating at $200 \mathrm{keV}$. FIG. 1 shows a BSE image of bone on the surface of the dental implant. A FIB cross-section of the bone/implant is shown in the BSE image in FIG. 2. Note the apparent osseointegration and growth of the bone into the coating pores. However, discontinuities in the bone/ polycrystalline coating interface are observed using bright field (BF) TEM imaging (see FIG. 3). Lattice fringes of the nanocrystalline mineralized grains within the bone are evident in the high resolution TEM image of FIG. 4. A high angle annular dark field (HAADF) STEM image of the metal/coating/bone interface region is shown in FIG. 5. Porosity in the ceramic coating, and especially at the coating/metal substrate is evident. The line in FIG. 5 corresponds to the position where an x-ray energy dispersive spectrometry (XEDS) profile shown was obtained (see FIG. 6). The profile indicates apparent interdiffusion between the bone and coating, inferring that chemical bonding occurs at this interface.

References

[1] J. Hermmerle, in Handbook of Histology Methods for Bone and Cartilage, eds. Y.H. An and K.L. Martin, Humana Press, Totowa, NJ (2003) 513.

[2] Introduction to Focused Ion Beams, eds. L.A. Giannuzzi and F.A. Stevie, Springer NY (2005). 


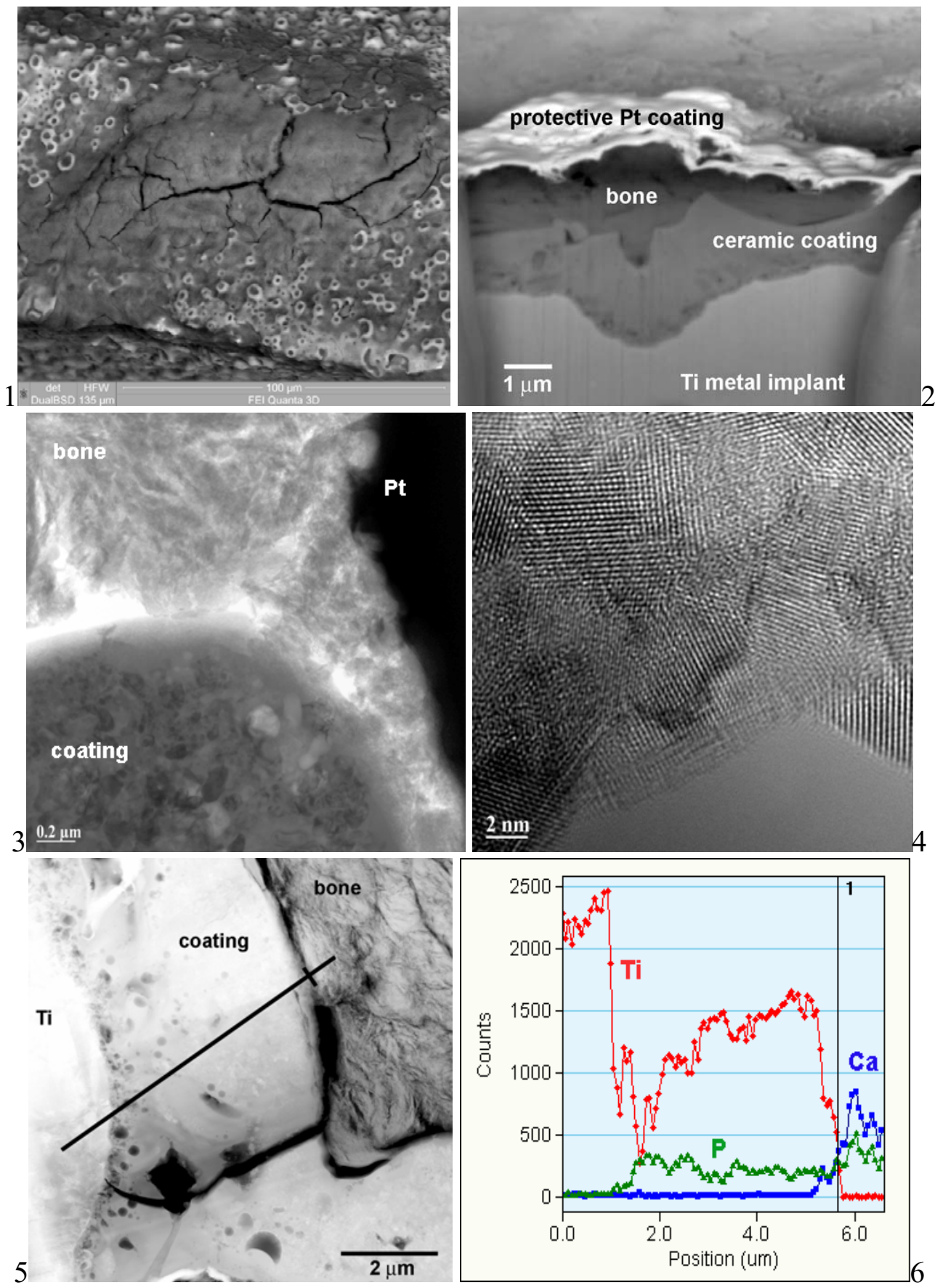

FIG. 1. BSE SEM image of bone on the implant surface.

FIG. 2. BSE SEM image the bone/coating/metal implant interfaces cross-sectioned by FIB.

FIG. 3. BF TEM image of the bone/coating region.

FIG. 4 HRTEM image of nanocrystalline mineralized grains in bone.

FIG. 5. HAADF STEM image of the metal implant/coating/bone specimen.

FIG. 6. XEDS profile across the metal/coating/bone interfaces. 